\title{
- Fluidoterapia em grandes animais. Parte I: agua corpórea, indicações e tipos de fluidos
}

\section{- Fluid therapy in large animals - Part I: body water, indications and fluid types}

Universidade Estadual
de Londrina - CCA - DCV
Caixa Postal 6001 - CEP: $86051-990$
Londrina-Paraná
Fone: (0**43) 371-4269/371-4329
End. Eletrôn.: dearoaco@uel.br

\section{Antônio Cézar de 0liveira Dearo - CRMV-PR - $\mathrm{n}^{0} 4428$}

Professor Assistente do Departamento de Clínicas Veterinárias da UEL/PR.

\section{RESUMO}

As alterações do equilíbrio hidroeletrolítico apresentam-se comumente associadas a diversas patologias envolvendo animais de grande porte. Com o objetivo de se promover a correção dessas alterações, é importante a elaboração de um plano de reposição hidroeletrolítica que, baseado nas informações da anamnese, do exame clínico e dos testes laboratoriais, deve incluir não só a escolha da solução mais adequada como também sua quantidade, via de administração e velocidade de reposição. Neste artigo são abordados aspectos referentes à fisiologia da água corpórea e eletrólitos, indicações da fluidoterapia e principais fluidos utilizados em Medicina Veterinária.

Palavras-chave: fluidoterapia, desidratação, eletrólitos, hidratação, fluidos.

\section{Distribuição da Água Corpórea e Eletrólitos}

$\underline{0}$ s desequilíbrios hidroeletrolíticos apresentam-se associados a inúmeras situações clínicas que acometem as grandes espécies domésticas. Considerando que a água e os eletrólitos orgânicos desempenham um papel essencial na manutenção das mais diversas funções do organismo animal, a correção desses desequilíbrios torna-se de vital importância para o sucesso do tratamento específico. Um exemplo clássico desse conceito pode ser representado por cavalos portadores de intenso desconforto abdominal agudo associado a enormes perdas de fluidos e eletrólitos e que requerem tratamento cirúrgico. Nessas situações, a não correção ou a correção inadequada dos desequilíbrios hidroeletrolíticos pode resultar no óbito do paciente antes mesmo do início da cirurgia.
Nas grandes espécies, aproximadamente $60 \%$ do peso corpóreo no adulto e $80 \%$ no neonato consiste de água, que se apresenta amplamente distribuída e em constante movimento entre os compartimentos intracelular (LI) e extracelular (LE) (MASON, 1972; SPURLOCK e FURR, 1990; REECE, 1991; GROSS, 1992; SCHMALL, 1997). O compartimento intracelular possui a maior quantidade da água corpórea e corresponde a $40 \%$ ou $2 / 3$ do peso total, ao passo que os $20 \%$ (1/3) restantes constituem o compartimento extracelular. Esse, por sua vez, é subdividido em compartimento plasmático (Lp) (5\%), intersticial (Li)(14\%) e transcelular (Lt) (1-6\%) (REECE, 1991; GROSS, 1992).

O líquido transcelular é representado pela água nas vias digestiva e urinária, no líquor, no humor aquoso, na cavidade peritoneal e no líqüido sinovial. Em herbívoros, especialmente em grandes ruminantes, a água contida no 
sistema digestório pode corresponder a até 10 a $15 \%$ do peso corpóreo (GROSS, 1992).

É importante ressaltar que esses valores representam mensurações aproximadas e que fatores como doença, clima, idade, estado nutricional e privação hídrica entre outros, resultam em diferentes valores de distribuição da água corpórea.

O constante movimento da água entre os compartimentos é decorrente de um gradiente osmótico estabelecido entre os dois lados da membrana celular. Em outras palavras, esse movimento é determinado pelo número de partículas osmoticamente ativas entre um lado e outro da membrana. Os principais íons responsáveis pela osmolaridade no LE e LI são o $\mathrm{Na}^{+} \mathrm{e} o$ $\mathrm{Cl}^{-}$, no LI, são o $\mathrm{K}^{+}$e o $\mathrm{PO}_{4}^{-}$(REECE, 1991; GROSS, 1992; SCHMALL, 1997). Desequilíbrios no balanço hídrico entre os compartimentos podem resultar de uma diminuição da ingestão, aumento das perdas de fluidos e eletrólitos ou, ainda, de uma combinação desses fatores. As alterações de volume dos compartimentos LI ou LE, produzidas em inúmeras condições clínicas, determinam mudanças nas respectivas pressões osmóticas com conseqüente redistribuição da água entre os compartimentos. Um exemplo prático desse conceito pode ser notado quando ocorre uma perda aguda de líqüidos, como na diarréia. $\mathrm{O}$ aumento da pressão osmótica resultante da perda hídrica determina o movimento de água do compartimento LI para o LE até que se estabeleça um novo eqüilíbrio osmótico (ROSE, 1981; SCHMALL, 1997).

O movimento contrário da água ocorre em situações de queimaduras onde há relativamente maior perda de eletrólitos do que água. $\mathrm{O}$ aumento da pressão osmótica do compartimento intracelular determina a passagem de água para o interior da célula causando edema (GROSS, 1992).

\section{Quando Instituir a Terapia Hídrica ?}

A reposição de fluidos tem como objetivos a restauração da volemia, a hidratação dos tecidos, a correção de desequilíbrios eletrolíticos e ácido-básicos, a estimulação de órgãos internos como, por exemplo, os rins, a administração de substâncias nutritivas (alimentação parenteral) e, além disso, o próprio fluido reposto também serve como meio para a administração de drogas (GROSS, 1992; FREESTONE, 1993).

Em grandes animais, as condições clínicas mais comuns que requerem fluidoterapia são representadas por diarréias, choque, síndrome cólica, desidratação após exercícios físicos extenuantes sob condições cli- máticas adversas (como por exemplo, no enduro eqüestre), ruptura de bexiga em potros, desequilíbrios metabólicos, obstruções ou rupturas esofágicas e doenças renais (SPIER et al., 1990; FREESTONE, 1993; BARTON e MOORE, 1999).

A maneira mais adequada de se determinar a necessidade e, ao mesmo tempo, elaborar o plano de reposição hidroeletrolítica, baseia-se na análise de informações precisas obtidas mediante a anamnese, o exame físico e, quando disponível, a realização de provas laboratoriais. Principalmente sob condições de campo, nas quais, na maioria das vezes, o recurso laboratorial não é disponível, o conhecimento preliminar dos mecanismos fisiopatológicos inerentes às mais variadas doenças é de fundamental importância para a elaboração lógica e correta do plano de reposição hidroeletrolítica.

Informamos sobre o tempo de evolução na doença, os tratamentos efetuados anteriormente, drogas, doses, intervalos de aplicação, sobre a ingestão de alimentos, a disponibilidade, qualidade e ingestão de água oferecida, a quantidade e a frequiência de micção e dos episódios de perdas eletrolíticas (diarréias, refluxo gastrointestinal colhido por sonda nasogástrica, entre outros) permitem uma noção preliminar acerca da gravidade de possíveis desequilíbrios (SPIER et al., 1990; FREESTONE, 1993). Um exemplo muito comum até os dias de hoje, e que exemplifica com clareza a importância desse questionamento, refere-se ao uso de diuréticos a exemplo da furosemida, administrados, de forma equivocada, por leigos, a cavalos com cólica. Considerando que a desidratação e a hipovolemia são fatores comumente associados à cólica eqüina e que, muitas vezes, são os responsáveis diretos pelo óbito de muitos cavalos, a perda de mais líqüidos induzida pela terapia diurética agravaria o problema.

Nos sinais clínicos normalmente relacionados às condições de perda hídrica, pode-se incluir a perda de peso, o aumento da frequiência cardíaca e do tempo de preenchimento capilar, a perda da elasticidade cutânea, o ressecamento das mucosas, a diminuição da temperatura em regiões de extremidades e a diminuição da produção de urina (SPIER et al., 1990; SCHMALL, 1992, 1997); contudo, a severidade desses sinais varia de acordo com os diferentes graus de desidratação. Porém, é interessante ressaltar que em situações clínicas nas quais a perda hídrica não é acompanhada de perdas eletrolíticas, o animal pode não apresentar sinais de desidratação mas, mesmo assim, estar desidratado.

$\mathrm{Na}$ prática, isso pode ser notado em animais que não se alimentam e não ingerem água e pode ser explicado pela modificação do gradiente osmótico estabelecido 


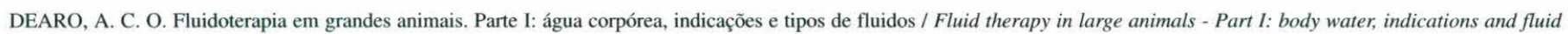
types. / Rev. educ. contin. CRMV-SP / Continuous Education Journal CRMV-SP, São Paulo, volume 4, fascículo 2, p. 3 - 8, 2001.

Tabela 1. Graus de desidratação.

\begin{tabular}{|c|c|c|c|c|c|}
\hline $\begin{array}{c}\text { Desidratação } \\
(\%)\end{array}$ & Classificação & $\begin{array}{c}\text { Turgor Cutâneo } \\
\text { (segundos) }\end{array}$ & $\begin{array}{c}\text { TPG* } \\
\text { (segundos) }\end{array}$ & VG (\%) & PPT (g/dl) \\
\hline $5-7$ & Leve & $2-3$ & $1-2$ & $40-50$ & $6.5-7.5$ \\
\hline $8-10$ & Moderada & $3-5$ & $2-4$ & $50-65$ & $7.5-8.5$ \\
\hline$>10$ & Severa & $>5$ & $>4$ & $>65$ & $>8.5$ \\
\hline
\end{tabular}

* TPC: Tempo de Perfusão Capilar

entre os compartimentos, onde o aumento da osmolaridade do LE determina a diminuição do volume hídrico do LI na tentativa de se restabelecer o equilíbrio (FREESTONE, 1993).

Do ponto de vista clínico, a desidratação pode ser subjetivamente classificada em leve, moderada e severa, de acordo com a gravidade dos sinais. Contudo, deve-se ter em mente que perdas fluidas inferiores a $5 \%$ do peso corpóreo não são manifestadas clinicamente (SCHMALL, 1992, 1997; FREESTONE, 1993; BARTON e MOORE, 1999). Em termos práticos, isso significa que nenhuma alteração clínica compatível com desidratação pode ser evidenciada em um cavalo adulto pesando $450 \mathrm{~kg}$ até que aproximadamente 201 de fluidos tenham sido perdidos.

A Tabela 1 mostra os diferentes graus de desidratação em função de algumas alterações clínicas e laboratoriais. Deve-se salientar que esses valores devem ser tomados apenas como guia geral e não como valores absolutos.

O volume globular (VG) ou hematócrito e a proteína plasmática total (PPT) são as duas mensurações laboratoriais mais comumente utilizadas para se avaliar o grau de desidratação (ROSE, 1981; SPURLOCK e WARD, 1990; SCHMALL, 1992, 1997). A elevação desses índices é indicativo de perda hídrica. Outras determinações como a uréia e a creatinina séricas também podem ser empregadas com essa finalidade, porém todos esses índices devem ser interpretados com cautela (SCHMALL, 1992, 1997). É importante que o VG e a PPT sejam analisados conjuntamente, pois, em algumas situações, a interpretação isolada desses valores pode resultar em erro. A estimulação simpática causada por condições de estresse ou excitação pode provocar a contração esplênica com liberação na circulação de mais hemácias (ROSE, 1981; SCHMALL, 1992, 1997). Nessas circunstâncias, há aumento do VG, não por perda fluida, mas, sim, pelo aumento do número hemácias não indicando, portanto, desidratação.

Em situações clínicas em que há perda de proteínas associada a patologias gastrointestinais como nas colites e peritonites ou em que há diminuição da produção decor- rente de problemas hepáticos, as proteínas plasmáticas podem apresentar-se diminuídas. Nessas situações, a PPT perde seu valor como indicador de desidratação (ROSE, 1981; MURRAY, 1992; SCHMALL, 1997).

Em potros e bezerros neonatos, os valores de VG e PPT podem variar em razão de diversos fatores, não sendo, portanto, indicadores fidedignos de desidratação (ROUSSEL e KASARI, 1990; SPURLOCK e FURR, 1990; FREESTONE, 1993).

Como já referido anteriormente, nem sempre as perdas hídricas estão associadas às perdas eletrolíticas, todavia, o que se observa na prática, é que a grande maioria dos desequilíbrios hídricos são acompanhados por desequilíbrios eletrolíticos e ácido-básicos em diferentes graus.

\section{Qual Fluido Deve ser Utilizado?}

As soluções de uso parenteral podem ser classificadas em colóides e cristalóides. Colóides são substâncias de alto peso molecular que, ao serem administradas, aumentam a pressão coloidosmótica intravascular e, com isso, estimulam a passagem de fluido do Li para o Lp. O dextran 70 e o plasma são os exemplos mais comuns de colóides sintéticos e naturais, respectivamente. Por questões de custo e pela possibilidade da ocorrência de efeitos colaterais associados à administração de colóides sintéticos, o uso dessas substâncias é extremamente restrito (SPURLOCK e WARD, 1990; SCHMALL, 1992; SEAHORN e CORNICK-SEAHORN, 1994).

$\mathrm{O}$ uso de plasma tem sua principal indicação em potros ou bezerros que não ingeriram de forma adequada o colostro e por isso apresentam falha na transferência da imunidade passiva. Outra indicação refere-se a situações clínicas em que a PPT encontra-se abaixo de $4 \mathrm{~g} / \mathrm{dl}$ em razão de perdas contínuas existentes (como nas colites) ou de extensa hemodiluição decorrente de prolongada fluidoterapia com cristalóides (MURRAY, 1992; SCHMALL, 1992, 1997).

Os cristalóides são as soluções mais amplamente empregadas em Medicina Veterinária. De maneira geral, são utilizadas quando se objetiva a correção de altera- 


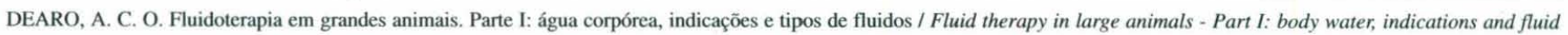
types. / Rev. educ. contin. CRMV-SP / Continuous Education Journal CRMV-SP, São Paulo, volume 4, fascículo 2, p. 3 - 8, 2001.

Tabela 2. Composição iônica, osmolaridade e tonicidade das principais soluções utilizadas em Medicina Veterinária.

\begin{tabular}{|l|c|c|c|c|c|c|c|c|}
\hline \multicolumn{1}{|c|}{ Solução } & $\begin{array}{c}\mathrm{Na}^{+} \\
(\mathbf{m E q} / \mathbf{l})\end{array}$ & $\begin{array}{c}\mathbf{K}^{+} \\
(\mathbf{m E q} / \mathbf{l})\end{array}$ & $\begin{array}{c}\mathbf{C r}^{+} \\
(\mathbf{m E q} / \mathbf{l})\end{array}$ & $\begin{array}{c}\mathbf{C a}^{++} \\
(\mathbf{m E q} / \mathbf{l})\end{array}$ & $\begin{array}{c}\mathbf{M g}^{++} \\
(\mathbf{m E q} / \mathbf{l})\end{array}$ & $\begin{array}{c}\mathbf{H C O}_{3} \\
(\mathbf{m E} / \mathbf{l})\end{array}$ & $\begin{array}{c}\text { Osm } \\
(\mathbf{m 0 s m} / \mathbf{l})\end{array}$ & Tonicidade \\
\hline Ringer c/Lactato & 131 & 4 & 110 & 3,6 & 0 & 28,5 & 274 & Isotônica \\
\hline $\mathrm{NaHCO}_{3} 5 \%$ & 600 & 0 & 0 & 0 & 0 & 600 & 1200 & Hipertônica \\
\hline $\mathrm{NaCl}$ 0,9\% & 154 & 0 & 154 & 0 & 0 & 0 & 308 & Isotônica \\
\hline Glicose 5\% & 0 & 0 & 0 & 0 & 0 & 0 & 253 & Isotônica \\
\hline $\mathrm{NaCl} 7,5 \%$ & 1200 & 0 & 1200 & 0 & 0 & 0 & 2400 & Hipertônica \\
\hline Plasma Eqüino & $136-145$ & $2,8-4,7$ & $98-108$ & $5,5-6,5$ & $1,4-2,2$ & $24-30$ & $270-300$ & Isotônico \\
\hline
\end{tabular}

ções de volume, de eletrólitos, dos níveis de energia e do equilíbrio ácido básico. A composição iônica das principais soluções utilizadas atualmente pode ser verificada na Tabela 2.

De forma ideal, o uso de cristalóides deve ter como base as análises laboratoriais dos diferentes eletrólitos séricos e gases sangüíneos, o $\mathrm{pH}$ e a tonicidade da solução. Entretanto, em razão da indisponibilidade da maior parte desses recursos para a grande maioria dos profissionais, o bom senso, embasado no conhecimento preliminar dos tipos de desequilíbrios mais comumente relacionados à situação clínica em questão, determinará a escolha da solução mais adequada.

A solução de Ringer com Lactato é a que apresenta composição mais próxima à do plasma (ROSE, 1981). É a solução empregada na maioria dos pacientes como fonte inicial e emergencial de reposição hidroeletrolítica até que as análises laboratoriais possam melhor direcionar a fluidoterapia (MASON, 1972; MORRIS, 1988). Deve ser utilizada em situações envolvendo perdas de fluidos e eletrólitos acompanhadas de acidose metabólica moderada. O lactato presente na solução funciona como fonte de base pela sua transformação em bicarbonato após metabolização hepática (MASON, 1972; HARTSFIELD, 1981; GROSS, 1992; BARTON e MOORE, 1999). É importante, contudo, ressaltar que distúrbios hepáticos intrínsecos, acidose metabólica severa $(\mathrm{pH}<7,1)$ ou estados de deficiência circulatória portal podem reduzir a biotransformação do lactato em bicarbonato e, com isso, reduzir o caráter alcalinizante da solução (HARTSFIELD, 1981; GROSS, 1992; BARTON e MOORE, 1999).

A solução de cloreto de sódio a $0,9 \%$ deve ser empregada em situações envolvendo estados de hipercalemia, hiponatremia, hipocloremia e alcalose metabóli- ca (GROSS, 1992; SEAHORN e CORNICK-SEAHORN, 1994). As situações clínicas mais comuns que requerem o uso de $\mathrm{NaCl} 0,9 \%$ são o uroperitônio em potros e a desidratação após exercícios físicos extenuantes com grandes perdas hidroeletrolíticas pelo suor (FREESTONE, 1993; BARTON e MOORE, 1999).

A hipercalemia observada em potros portadores de uroperitônio contra-indica o uso de soluções contendo potássio, como, por exemplo, a solução de Ringer ou Ringer com Lactato.

A sudorese intensa observada em eqüinos submetidos a provas eqüestres de longas distâncias, com predomínio de metabolismo aeróbio como nos enduros, e promovidas notadamente em dias de altas temperaturas, determina uma grande depleção das reservas hidroeletrolíticas (CARLSON, 1987; ECKER, 1995; GEOR e McCUTCHEON, 1996). Nessas situações, as perdas hídricas podem alcançar valores superiores a 40 litros por prova. Grandes quantidades de sódio, cloreto, potássio, cálcio e magnésio são perdidas pelo suor; entretanto, os íons sódio e cloreto são aqueles perdidos em maior quantidade (CARLSON, 1987; ECKER, 1995). A perda excessiva de cloreto determina a mobilização de grandes quantidades de bicarbonato pelo organismo, resultando, dessa forma, num quadro de alcalose metabólica hipoclorêmica (CARLSON, 1987; ROSE, 1987; FREESTONE, 1993; SCHMALL, 1997). Por essa razão, deve-se tomar muito cuidado com a utilização de soluções contendo bicarbonato para a correção de desequilíbrios eletrolíticos e ácido básicos provocados por exercícios intensos, especialmente em provas de longas distâncias.

A solução de Ringer deve ser utilizada em situações envolvendo déficits de fluido e eletrólitos acompanhados de alcalose metabólica (GROSS, 1992; BARTON e MOORE, 1999). 
DEARO, A. C. O. Fluidoterapia em grandes animais. Parte I: água corpórea, indicações e tipos de fluidos / Fluid therapy in large animals - Part I: body water, indications and fluid types. / Rev. educ. contin. CRMV-SP / Continuous Education Journal CRMV-SP, São Paulo, volume 4, fascículo 2, p. 3 - 8, 2001.

A solução de glicose a $5 \%$ deve ser empregada em situações caracterizadas por diminuição das reservas energéticas (hipoglicemia) e desequilíbrios primariamente hídricos e não eletrolíticos, ou seja, nos casos em que a desidratação é hipertônica (SEAHORN e CORNICKSEAHORN, 1994). Na prática isso pode ser encontrado em animais que não estejam se alimentando e nem ingerindo água. Seu uso também é indicado em potros ou bezerros neonatos que não estejam ingerindo leite e conseqüentemente necessitem suporte energético (SCHMALL, 1992,1997).

As soluções de bicarbonato de sódio têm como indicação a correção de estados de acidose metabólica severa caracterizados por $\mathrm{pH}$ inferior a 7,2 ou déficit de base superior a $10 \mathrm{mEq} / \mathrm{l}$ (ROSE, 1981; SEAHORN e CORNICK-SEAHORN, 1994; SCHMALL, 1997; BARTON e MOORE, 1999). Essa recomendação baseia-se no fato de que quadros de acidose metabólica de menor gravidade podem ser freqüentemente solucionados pela correção da perfusão tecidual por meio de soluções eletrolíticas como a de Ringer com Lactato e pela correção da causa primária desencadeante do desequilíbrio ácidobásico.

Soluções de bicarbonato de sódio não devem ser misturadas a outras soluções contendo cálcio, uma vez que a formação de um precipitado insolúvel de carbonato de cálcio inviabiliza seu uso (HARTSFIELD, 1981; KEMP, 1988; SEAHORN e CORNICK-SEAHORN, 1994; BARTON e MOORE, 1999). Sua administração deve ser cuidadosa e com base nos valores da hemogasometria, pois a administração em excesso pode provocar alcalose metabólica de difícil reversibilidade e depressão respiratória (SCHMALL, 1992, 1997).
A solução hipertônica de cloreto de sódio a 7,5\% (2400 mOsm/1) utilizada em situações emergenciais na recuperação volêmica de pacientes em choque hipovolêmico, hemorrágico e séptico vem ganhando considerável espaço na Medicina Veterinária nos últimos anos (BERTONE et al., 1990; BERTONE, 1991; JEAN, 1998). Por se tratar de uma solução hipertônica, seu mecanismo de ação envolve a passagem de fluidos do LI e Li para o Lp, aumentando, dessa forma o volume circulante (SCHMALL, 1997; JEAN, 1998; STEWART, 1998). Pesquisas realizadas em pacientes humanos e animais destacam entre os inúmeros benefícios a melhoria do rendimento cardíaco, da pressão arterial, do fluxo plasmático renal e a diminuição da resistência vascular periférica e pulmonar (VELASCO e SILVA, 1989; BERTONE et al., 1990).

A solução hipertônica de $\mathrm{NaCl}$ a 7,5\% deve ser utilizada somente em situações emergenciais nas quais se requer uma rápida expansão de volume circulatório (BERTONE, 1991, 1998; JEAN, 1998). A rápida expansão volêmica permite a manutenção temporária das funções orgânicas até que medidas ou ações definitivas sejam tomadas para interromper a causa primária do choque. Nesse contexto, vale dizer que seu uso é questionável em situações de hemorragias graves que não possam ser controladas por meio das manobras usuais de hemostasia (BERTONE, 1991; JEAN, 1998). É importante lembrar que seus efeitos são temporários, com duração entre 90 a 120 minutos (SCHMALL, 1997), e que a continuidade da expansão volêmica por meio de soluções eletrolíticas após a administração de $\mathrm{NaCl}$ a $7,5 \%$ é condição indispensável para a correção do desequilíbrio circulatório e hídrico (SPURLOCK e WARD, 1990; SCHMALL, 1992, 1997; BERTONE, 1998).

\section{SUMMARY}

Fluid and electrolyte imbalances have been related to several disorders encountered in large animals. In order to correct such imbalances, a fluid therapy plan including the fluid type, amount, route of administration and flow rates must be set up. This article describes some aspects related to body water and electrolyte physiology, fluid therapy indications and the fluids commonly used in veterinary medicine.

Key words: body water, dehydration, electrolytes, hydration, fluids. 


\section{REFERÊNCIAS}

1. BARTON, M. H.; MOORE, J. N. Fluid and electrolyte therapy. In: COLAHAN, P. T.; MAYHEW, I. G.; MERRITT, A. M. et al. Equine medicine and surgery. 5. ed. St. Louis: Mosby, 1999. cap. 4, p. 146-152.

2. BERTONE, J. J. Hypertonic saline in management of shock. In: REED, S. M.; BAYLY, W. M. Equine internal medicine. Philadelphia: W. B. Saunders, 1998. cap. 4, p. 198-201.

3. BERTONE, J. J. Hypertonic saline in the management of shock in horses Compendium on Continuing Education for the Practicing Veterinarian, v. 13, n. 4, p. 665-668, 1991.

4. BERTONE, J. J.; GOSSETT, K. A.; SHOEMAKER, K. E. et al. Effect of hypertonic vs isotonic saline on responses to sublethal Escherichia coli endotoxemia in horses. American Journal of Veterinary Research, v. 51, n. 7, p. 999-1007, 1990.

5. CARLSON, G. P. The exhausted horse syndrome. In: ROBINSON, N. E. Current therapy in equine medicine. 2. ed. Philadelphia: W. B. Saunders, 1987. cap. 12, p. 482-485.

6. ECKER, G. L. Fluid and ion regulation: a primer on water and ion losses during exercise. Equine Veterinary Education, v. 7, n. 4, p. $210-215,1995$.

7. FREESTONE, J. F. Fluid therapy: an integral part of treating common equine disorders. Veterinary Medicine, v. 88, n. 6, p. $563-570,1993$.

8. GEOR, R. J.; McCUTCHEON, L. J. Thermoregulation and clinical disorders associated with exercise and heat stress. Compendium on Continuing Education for the Practicing Veterinarian, v. 18, n. 4, p. 436-445, 1996.

9. GROSS, D. R. Drogas que atuam no equilíbrio líquido e eletrolítico. In: BOOTH, N. H.; MCDONALD, L. E. Farmacologia e terapêutica em veterinária. 6 . ed. Rio de Janeiro: Guanabara Koogan, 1992. cap. 8, p. 427-438.

10. HARTSFIELD, S. M. Sodium bicarbonate and bicarbonate precursors for treatment of metabolic acidosis. Journal of the American Veterinary Medical Association, v. 179, n. 9, p. 914916, 1981.

11. JEAN, K. Y. Clinical hypertonic saline solutions. In: WHITE, N. A; MOORE, J. N. Current techniques in equine surgery and lameness. 2. ed. Philadelphia: W. B. Saunders, 1998. cap. 4, p. $15-19$.

12. KEMP, D. T. A comprehensive guide to intravenous fluid therapy in horses. Veterinary Medicine, v. 83, n. 2, p. 193-212, 1988.

13. MASON, T. A. A practical approach to fluid therapy in the horse. Australian Veterinary Journal, v. 48, n. 12, p. 671-676, 1972.
14. MORRIS, D. D. Medical therapy of colic. In: GORDON, B. J.; ALLEN JR., D. Field guide to colic management in the horse. Lenexa: Veterinary Medicine, 1988. cap. 12, p. 201-206.

15. MURRAY, M. J. Acute colitis. In: ROBINSON, N. E. Current therapy in equine medicine. 3. ed. Philadelphia: W. B. Saunders, 1992. cap. 5, p. 244-250.

16. REECE, W. O. Body water. In: O. Physiology of domestic animals. Malvern: Lea \& Febiger, 1991. p. 8190.

17. ROSE, R. J. Fluid, electrolyte and acid-base disturbances associated with exercise. In: ROBINSON, N. E. Current therapy in equine medicine. 2. ed. Philadelphia: W. B. Saunders, 1987. cap. 12 , p. $479-481$.

18. ROSE, R. J. A physiological approach to fluid and electrolyte therapy in the horse. Equine Veterinary Journal, v. 13, n. 1, p. 7-14, 1981.

19. ROUSSEL JR., A. J.; KASARI, T. R. Using fluid and electrolyte therapy to help diarrheic calves. Veterinary Medicine, v. 85, n. 3, p. 303-311, 1990.

20. SEAHORN, T. L.; CORNICK-SEAHORN, J. Fluid therapy. The Veterinary Clinics of North America. Equine Practice, v. 10 , n. 3 , p. $517-525,1994$.

21. SCHMALL, L. M. Fluid and electrolyte therapy. In: ROBINSON, N. E. Current therapy in equine medicine. 3. ed. Philadelphia: W. B. Saunders, 1992. cap. 1, p. 18-22.

22. SCHMALL, L. M. Fluid and electrolyte therapy. In: ROBINSON, N. E. Current therapy in equine medicine. 4. ed. Philadelphia: W. B. Saunders, 1997. cap. 16, p. 727-731.

23. SPIER, S. J.; SNYDER, J. R.; MURRAY, M. J. Fluid and electrolyte therapy for gastrointestinal disorders. In: SMITH, B. P. Large animal internal medicine. St. Louis: Mosby, 1990. cap. 30 , p. $708-715$.

24. SPURLOCK, S. L.; FURR, M. Fluid therapy. In: KOTERBA, A. M.; DRUMMOND, W. H.; KOSCH, P. C. Equine clinical neonatology. Malvern: Lea \& Febiger, 1990. cap. 31, p. 671700 .

25. SPURLOCK, S. L.; WARD, M. V. Fluid therapy for acute abdominal disease. In: WHITE, N. A The Equine acute abdomen. Malvern: Lea \& Febiger, 1990. cap. 6, p. 160-172.

26. STEWART, R. H. Considerations in fluid and electrolyte therapy. In: REED, S. M.; BAYLY, W. M. Equine internal medicine. Philadelphia: W. B. Saunders, 1998. cap. 4, p. 192-198.

27. VELASCO, I. T.; SILVA, M. R. Choque hipovolêmico: ressuscitação hipertônica. Atualização Cardiológica, v. 5, n. 6, p. 3-9, 1989. 